\title{
Mechanochemical reaction kinetics scales linearly with impact energy
}

\author{
Leonarda Vugrin, ${ }^{\mathrm{a}, \perp}$ Maria Carta, ${ }^{\mathrm{b}, \perp}$ Stipe Lukin, ${ }^{\mathrm{a}}$ Ernest Meštrović, ${ }^{\mathrm{c}}$ Francesco Delogu, ${ }^{\mathrm{b}, *}$ \\ Ivan Halasz ${ }^{\mathrm{a}, *}$
}

${ }^{a}$ Ruđer Bošković Institute, Bijenička c. 54, 10000 Zagreb, Croatia

${ }^{b}$ Department of Mechanical, Chemical and Materials Engineering, University of Cagliari, via Marengo 2, 09123 Cagliari, Italy

${ }^{\mathrm{c}}$ Department of Chemistry, Faculty of Science, University of Zagreb, Horvatovac 102a, 10000 Zagreb, Croatia

${ }^{\perp}$ equal contribution

Email: francesco.delogu@unica.it, ivan.halasz@irb.hr

\begin{abstract}
Inelastic collisions of the milling media in ball milling provide energy to the reaction mixture required for a chemical transformations. However, movement of the milling media results also in physical mixing of reactants, which too may enable a chemical reaction. Separating the two contributions is challenging and gaining a direct insight into the purely mechanochemically driven reactivity is accordingly hindered. Here, we have applied in situ reaction monitoring by Raman spectroscopy to a suitable, purely mechanically activated, chemical reaction and combined kinetic analysis with numerical simulations to access experimentally unattainable milling parameters. The breadth of milling conditions allow us to establish a linear relationship between the reaction rate and the energy dose received by the sample. Consequently, different kinetic profiles in time scale to the same profile when plotted against the energy dose, which increases with the ball mass, the average ball velocity and the frequency of impacts, but it decreases with the hardness of the milling media due to more elastic collisions. The fundamental relationship between kinetics and energy input provides the basis for planning and optimisation of mechanochemical reactions and is essential for transferability of mechanochemical reactions across different milling platforms.
\end{abstract}




\section{Introduction}

Mechanochemical processing of solids, in particular by ball milling, has become a recognized alternative to the traditional solution-based approach to chemical synthesis. ${ }^{1}$ Until the end of 1970 s it has mostly been confined to mineral extraction and powder metallurgy, but is now rapidly expanding into all areas of synthesis and materials preparation and is playing a crucial role in the ongoing quest for advanced materials. ${ }^{2-5}$ Presently, mechanochemical methods stand as one of green alternatives to solution reactions, ${ }^{6,7}$ as they not only significantly reduce or even eliminate the use of solvents, but in fact fulfil all 12 principles of green chemistry. ${ }^{8}$ Notably, by not relying on dissolution, mechanochemistry enables the use of insoluble precursors, ${ }^{9}$ while the very nature of exerting a mechanical force on the reaction mixture has recently enabled remarkable reactivity by using piezoelectric materials. ${ }^{10,11}$

With prospects of wider application in large-scale manufacturing, ${ }^{12,13}$ better understanding of fundamental aspects of mechanochemical reactions is becoming increasingly important. With the recent insights on atomic and molecular exchange and energy-controlled reaction paths, ${ }^{14,15}$ understanding of the energetics of milling, ${ }^{16,17}$ and molecular-level interaction of compressing particles, ${ }^{18}$ it is becoming of paramount importance to understand the fundamental aspect of energy transfer during ball collisions. Particularly since the use of different milling vials, balls and frequency profoundly effects the reaction yield for "energetically challenging" reactions ${ }^{16}$ and has, on occasion, altered product selectivity. ${ }^{19}$ Although investigation on this subject has been carried out for metals and ceramic materials, ${ }^{20}$ clear experimental evidence is decidedly lacking for softer inorganic and organic reactions, ${ }^{21-24}$ dominantly due to the difficulties associated with separating the mechanically activated contribution from ageing ${ }^{25}$ in the overall reaction.

Here, we systematically vary the rate of energy transfer to the sample and monitor how it affects reaction kinetics. To this aim, we have found a suitable model reaction in the trimerisation of bis(dibenzoylmethanato) nickel(II) $\left(\mathrm{Ni}(\mathrm{dbm})_{2}\right.$, Scheme 1$) .{ }^{26}$ In the brown $\mathrm{Ni}(\mathrm{dbm})_{2}$, nickel exhibits a square planar coordination with two apical coordination sites free. The green trimer, $\left[\mathrm{Ni}(\mathrm{dbm})_{2}\right]_{3}$, is formed via bridging oxygen atoms resulting in an octahedral coordination around nickel. Having a single phase as the reactant, trimerisation is independent of mixing, while the trimer is the sole product that, apparently, forms in a single reaction step. The trimerisation occurs also by heating, but above $200{ }^{\circ} \mathrm{C},{ }^{26}$ which is a temperature that is never reached on the bulk in a vibratory mixer mill. ${ }^{27,28}$ The reactant and the product are both stable solids and the reaction mixture is throughout milling in the form of a free-flowing powder enabling reaction monitoring by in situ Raman spectroscopy. Kinetic profiles were described by a 
model that accounts for the statistical nature of ball milling, ${ }^{29,30}$ and the kinetic modelling was supported by numerical simulations of the milling process.

Scheme 1. Molecular structures in the solid state of (a) the brown reactant $\mathrm{Ni}(\mathrm{dbm})_{2}$ and (b) the green product $\left[\mathrm{Ni}(\mathrm{dbm})_{2}\right]_{3}{ }^{26}$ (c) Photographs of the reactant (top) and the product (bottom). Colour code: green -nickel, red - oxygen, black - carbon, and white - hydrogen. Top view and side view in a). Hydrogen atoms omitted in b). Molecular diagrams were prepared using PLATON. ${ }^{31}$

a)

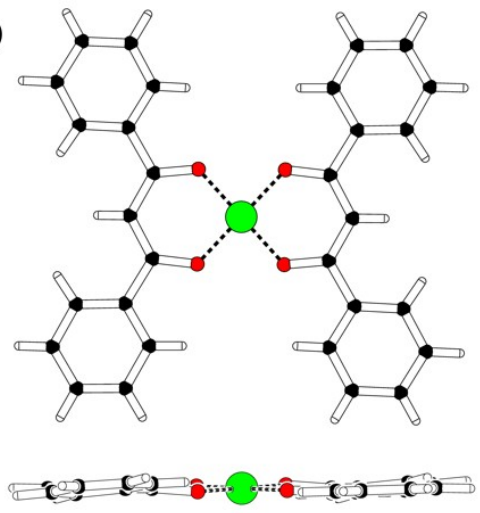

b)

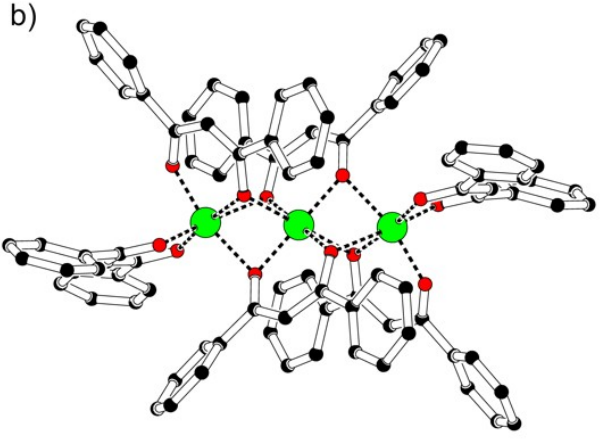

c)

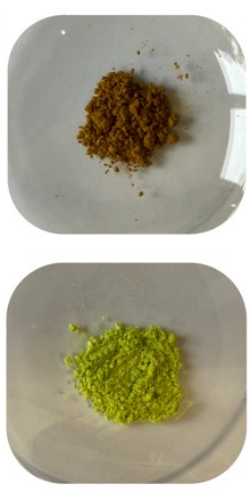

\section{Results and discussion}

Variation of energy input was here achieved by the variation of the ball mass, the ball size and the milling frequency. Milling experiments were conducted always with one milling ball that was made of one of 4 different materials (agate, stainless steel (SS), zirconia $\left(\mathrm{ZrO}_{2}\right)$ or tungsten carbide (WC)), was of two diameters (8 or $10 \mathrm{~mm}$ ) and at four frequencies (27.5, 30.0, 32.5 or $35.0 \mathrm{~Hz}$ ) (Table 1). Reaction profiles for kinetic analysis were extracted from in situ Raman spectroscopy monitoring (Fig. 1), ${ }^{32}$ where each in situ collected spectrum was represented as a linear combination of the reactant and the product spectra. Each spectrum was thus decomposed into fractional contributions from the two components, reactant and product, and, when performed for a series of in-situ-collected spectra, enabled the construction of reaction profiles (Figs. 2 and S1-S3).

Table 1. The explored milling conditions comprising 6 different milling balls, each used at 4 frequencies. A tungsten carbide ball of $10 \mathrm{~mm}$ and an agate ball of $8 \mathrm{~mm}$ were unavailable. 


\begin{tabular}{|c|c|c||c|}
\hline \multirow{2}{*}{$\begin{array}{c}\text { Ball } \\
\text { material }\end{array}$} & \multicolumn{2}{|c||}{ Ball diameter / mm } & \multirow{2}{*}{ Frequency / Hz } \\
\cline { 2 - 4 } & 8.0 & 10.0 & \\
\hline SS & 2.0 & 4.0 & 35.0 \\
\hline ZrO2 & 1.6 & 3.5 & 32.5 \\
\hline WC & 3.9 & & 30.0 \\
\hline Agate & & 1.5 & 27.5 \\
\hline
\end{tabular}
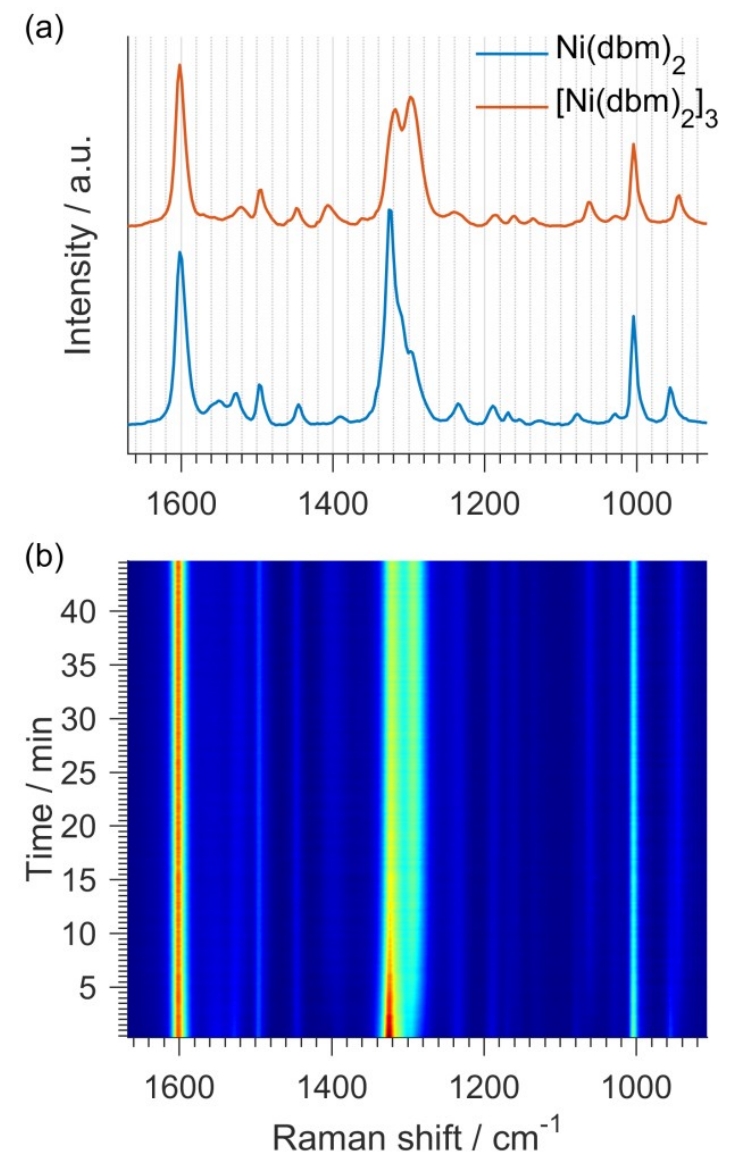

Figure 1. (a) Normalized Raman spectra of the reactant $\mathrm{Ni}(\mathrm{dbm})_{2}$ and the product $\left[\mathrm{Ni}(\mathrm{dbm})_{2}\right]_{3}$ used in a least-squares procedure for extracting reaction profiles. (b) Time-resolved 2D plot of an example reaction with one $10 \mathrm{~mm}$ stainless steel ball at a milling frequency of $32.5 \mathrm{~Hz}$. 
In all experiments, the reactant fraction, $\alpha_{R}(t)$, decreases smoothly with the duration of milling and, in general, the mechanochemical reaction is faster at higher milling frequency and when heavier balls are used (Figs. 2a, and S1-S3). In rationalizing the observed kinetic curves, we first consider the reactions performed with the same ball, but at different milling frequencies. We first note that the reaction times of the datasets collected at different milling frequencies can be suitably rescaled to make the kinetic curves overlap to a remarkable extent by multiplying the reaction time with a scaling factor $\phi$, which then defines the rescaled time $t_{s c}=\phi t$ (Fig. 2b). The $\phi$ coefficients exhibit a marked dependence on the milling frequency, $f$. Specifically, the $\phi$ coefficients arrange according to approximately linear trends when plotted as a function of the third power of $f$, as in Fig. 2c.
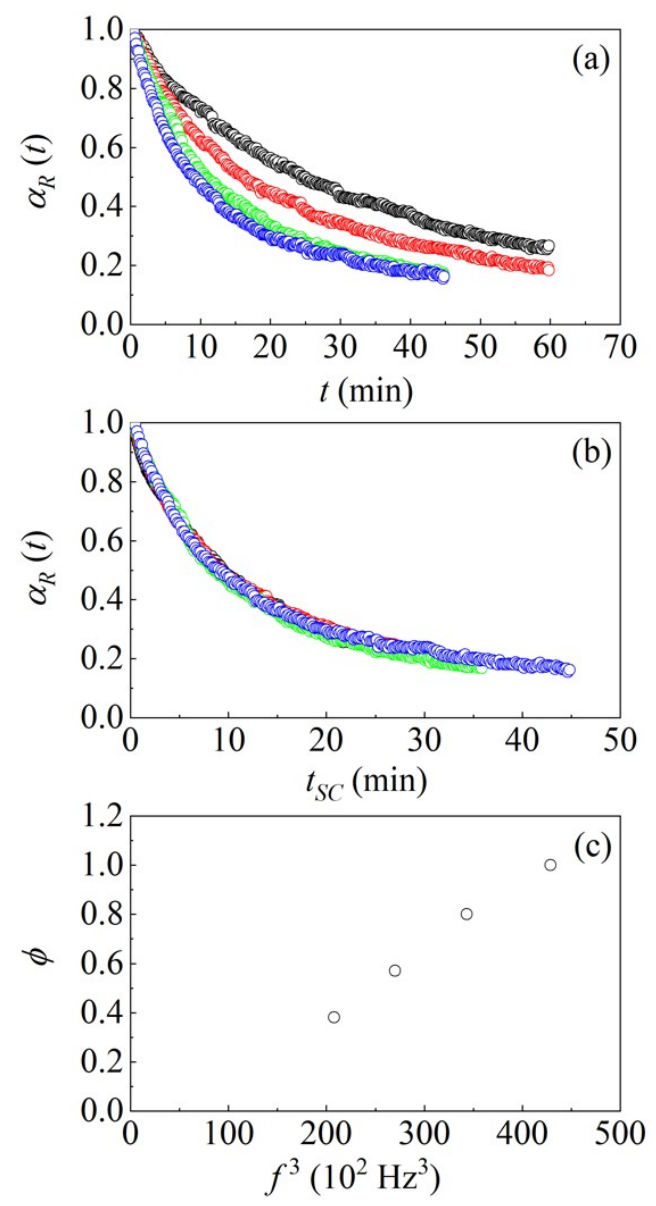

Figure 2. (a) The reactant fraction $\alpha_{R}(t)$, as a function of time, $t$ for milling experiments at four milling frequencies and using one stainless steel milling ball of $8 \mathrm{~mm}$ in diameter. (b) The reactant fraction $\alpha_{R}(t)$, 
as a function of the rescaled time, $t_{S c}$. (c) The dependence of the scaling parameter, $\phi$, with the third powder of the milling frequency. See text for details.

Such a behaviour indicates that the chemical reactions at different milling frequencies are dependent on a single processing parameter affecting the reaction rate. Indeed, scaling of the reaction kinetics with the third power of the milling frequency will be characteristic for a purely mechanically activated mechanochemical reaction where the kinetics is determined by the energy received by the sample from the milling ball impacts. Considering that the number of impacts per unit time, $N$, is proportional to milling frequency, $N \sim f$, and that, on average, each impact imparts an energy $E$ on the small amount of powder residing between the jar wall and the ball during an impact, the energy imparted on the powder per unit time will be equal to $E N$. This is the energy dose received by the sample in unit time. Since $E$ is proportional to the kinetic energy of the colliding milling ball, and the velocity of the ball is proportional to the milling frequency $f, E$ is proportional to $f^{2}$ (Fig. S30). Consequently, the energy dose received by the sample per unit time is proportional to $f^{3}$,

$E N \sim v^{2} f \sim f^{2} f=f^{3}$.

The reaction profiles at different frequencies should thus collapse on the same curve if plotted against the energy dose received. Such a behaviour of the observed reaction profiles provides further support for our model reaction to be a purely mechanically activated process where impacts, i.e. the impulsive transfers of mechanical energy from the ball to the powder, drive the mechanochemical transformation.

\section{The kinetic analysis}

In the kinetic model applied here, we assume that impacts generate mechanical stress within the volume of trapped powder and at contact surfaces between particles contained within the trapped volume, causing plastic deformation. We also assume that local mechanical stresses induce the establishment of the so-called critical loading conditions (CLCs) in irregularly distributed small volumes $v^{i}$ of the sample that is experiencing a ball impact (Scheme 2). It is within volumes $v^{i}$ that the conditions for the reactant to transform into the product are met. 


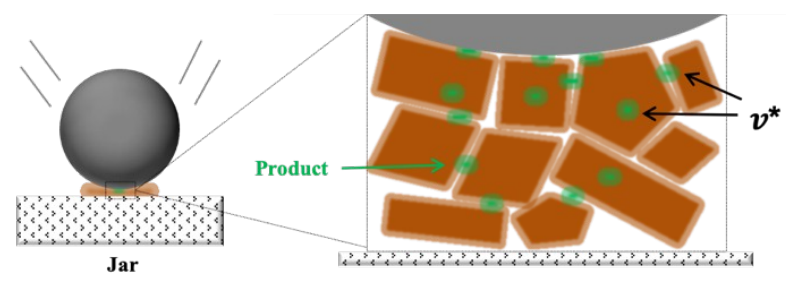

Scheme 2. Representation of a ball collision with the material trapped between the reaction jar wall and the ball. Within the trapped sample, randomly distributed small volumes $v^{i}$ (depicted in green) experience critical loading conditions (CLC) that enable the formation of products.

The involvement of the powdered sample in impacts is stochastic, meaning that the distribution of volumes $v^{i}$ within the whole of the sample is random. It may happen that the volumes $v^{i}$ experience CLCs more than once and coexist within the total volume $V$ of the powder sample. In general, volumes $v^{i}$ involved in different number of CLCs can be expected to exhibit a different product molar fraction, $\alpha_{P, i}$. Then, the global kinetics of the mechanically activated transformation can be rationalized once local kinetics is combined with a suitable statistical framework.

To this aim, we assume that (i) vigorous stirring keeps powder charge homogeneous, (ii) consecutive impacts are independent of each other, (iii) impacts can involve any given volume fraction of powder charge with the same probability, and (iv) CLCs generated by individual ball impacts always affect the same number of individual volumes $v^{i}$ per impact. The summed volumes $v^{i}$ from a single impact lead to the volume fraction of the powder that experiences CLC during a single impact:

$$
\kappa=v / V
$$

Transformation rates typically suggest that the volume $v$ of powder affected by CLCs during a single impact is significantly smaller than the total volume of the powder, $V$, and $\kappa$ can be expected to be much smaller than 1.

Statistical considerations allow expressing the volume fraction of powder affected by CLCs $i$ times after a total of $m$ impacts experienced by the whole powder as

$$
\chi_{i}(m)=\left[(\kappa m)^{i} / i !\right] \exp (-\kappa m)
$$

Eq. 2 fulfils the condition 


$$
\sum_{i=0}^{\infty} X_{i}(m)=1
$$

To relate the global kinetics of the mechanochemical transformation to the local kinetics in individual volumes $v^{i}$, we assume that any volume fraction of powder that has undergone $i$ CLCs after $m$ impacts, $\chi_{i}(m)$, can be associated with a specific value of the product molar fraction, $\alpha_{P, i}$. Thus, the global product volume fraction after $m$ impacts can be expressed as

$$
\alpha_{P}(m)=\sum_{i=1}^{\infty} X_{i}(m) \alpha_{P, i}
$$

Combining the statistical and chemical factors involved in the mechanical processing by ball milling, Eq. 4 describes the global chemical transformation kinetics. Taking into account that the total number of impacts, $m$, experienced by the powder sample can be related to the product of milling frequency, $f$, and time, $t$, the Eq. 2 can be rewritten in the time-dependent form

$$
X_{i}(t)=\left[(\kappa f t)^{i} / i !\right] \exp (-\kappa f t)=\left[(k t)^{i} / i !\right] \exp (-k t)
$$

Where, with $k=\kappa f$, we now have the volume fraction of the powder affected by CLCs per unit time. The Eq. 4 can now be rewritten as

$$
\alpha_{P}(t)=\sum_{i=1}^{\infty} X_{i}(t) \alpha_{P, i}
$$

The Eq. 6 can be used to best-fit the experimental reaction profiles once the dependence of $\alpha_{P, i}$ on the number $i$ of CLCs is known, i.e. the local kinetics is properly described. In this respect, we note that the simplest local kinetics corresponds to a complete reaction within the volume $v$ after a single CLC. This translates into the exponential law

$$
\alpha_{R}(t)=\exp (-k t)
$$

where $\alpha_{R}(t)$ is the reactant volume fraction. Accordingly, the volume fraction of the products can be expressed as

$$
\alpha_{P}(t)=1-\exp (-k t)
$$


However, it is evident from Fig. 3 that the semi-logarithmic plot of the reactant volume fraction, $\alpha_{R}(t)$, is not linear and, hence, the reaction kinetics cannot be described by a simple exponential given in the Eq. 7.

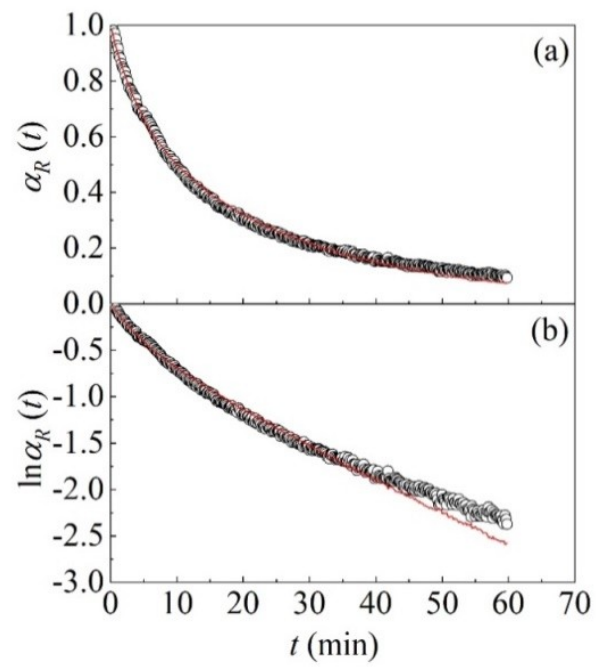

Figure 3. (a) The reactant fraction, $\alpha_{R}(t)$, and (b) the logarithm of the reactant fraction, $\alpha_{R}(t)$, as a function of the milling time, $t$. Data refer to experiments carried out with a $10-\mathrm{mm} \mathrm{ZrO}_{2}$ ball at $30.0 \mathrm{~Hz}$. Best-fitted curves are shown.

In contrast, a satisfactory best fitting can be obtained by assuming that the volume fraction of powder affected by CLCs per unit time, $k$, varies with the reactant volume fraction, $\alpha_{R}(t)$, as follows:

$$
k=k_{R} \alpha_{R}(t)+k_{P}\left[1-\alpha_{R}(t)\right] .
$$

The best fitting was performed taking advantage of the experimental $\alpha_{R}(t)$ datasets and using the $k_{R}$ and $k_{P}$ values as fitting parameters. The resulting curves, shown in Figs. S4-S27, display a satisfactory agreement in all the different cases investigated, which provides considerable support to the hypothesis leading to Eq. 9.

Eq. 9 stipulates that the volume fraction of the powder affected by CLCs per unit time, $k$, changes during the course of the transformation. In particular, $k$ varies linearly with $\alpha_{R}(t)$ between a value characteristic of the reactant phase, $k_{R}$, and a value characteristic of the product phase, $k_{P}$. At the 
beginning of the reaction, when $\alpha_{R}=1, k=k_{R}$, whereas $k=k_{P}$ once that the reactant phase has reacted completely and $\alpha_{R}=0$.

In rationalizing why the constant $k$ changes gradually from $k_{R}$ to $k_{P}$, we recall the Eq. 1 . It expresses the volume fraction of powder subjected to CLCs per impact as the ratio between the volume of powder affected by CLCs, $v$, and the total volume of powder, $V$. Since the product is denser than the reactant, ${ }^{26}$ the total volume occupied by the powder, $V$, decreases as the transformation takes place. Therefore, we should expect $k$ to increase. This means that $k_{P}$ should be larger than $k_{R}$, and, accordingly, we should observe an increasingly faster kinetics. In contrast, we observe that the reaction slows down as it advances and that the initial constant, $k_{R}$, is larger than the final, $k_{P}$. In other words, the formation of the product makes the reaction slower.

Since this behaviour cannot be related to a change in density between the reactant and the product, we hypothesize that it stems from a change in the mechanical properties of the milled powder as the amount of the product increases. In particular, we recall that, for metals and ceramics subjected to ball milling, the amount of powder affected by CLCs during individual impacts decreases as the hardness of the milled material increases. ${ }^{33,34}$ Based on such evidence, our results suggest that the hardness of the product is greater than the hardness of the reactant, causing the volume subjected to CLCs per impact, $v$, to decrease more than $V$, which is decreasing with the difference in densities of the reactant and the product. This would readily explain why $k_{R}$ is larger than $k_{P}$.

Given the definition of $\kappa$ (Eq. 1) and, since $k=\kappa f$, the values of $k_{P}$ and $k_{R}$ mirror the quantity of the powder affected by CLCs in each impact. Both $k_{P}$ and $k_{R}$ increase as the milling frequency increases and accordingly, the the impact energy and the frequency of impacts. Milling ball velocity during mechanochemical processing is hardly accessible experimentally over a longer period of time. We have thus used numerical simulations to estimate the average ball velocity as it collides with the reaction chamber walls and the amount of energy imparted to the sample upon each impact. Energy transfer during the collision will be dependent on the mechanical properties of the colliding materials. Since the vessel materials was always the same, the hardness of the ball material will affect the energy transfer during impacts. To take into account this variation in numerical simulations, we have assumed different values of the restitution coefficient, $e$, for different ball materials, which take into account how much a single impact differs from an ideal case of a fully elastic collision. Specifically, a restitution coefficient value of 1 means complete restoration of the kinetic energy of the ball and an elastic collision. On the contrary, an $e$ value of 0 means that the impact is inelastic and that the ball effectively comes to a stop upon impact. 
If our studied reaction is dependent on the impact energy, the $k_{P}$ and $k_{R}$ values, utilized to best fit the experimental kinetic curves, must show a marked dependence on the impact energy, $E$. Indeed, a linear dependence is observed, as shown in Fig. 4 for all the monitoring reactions.
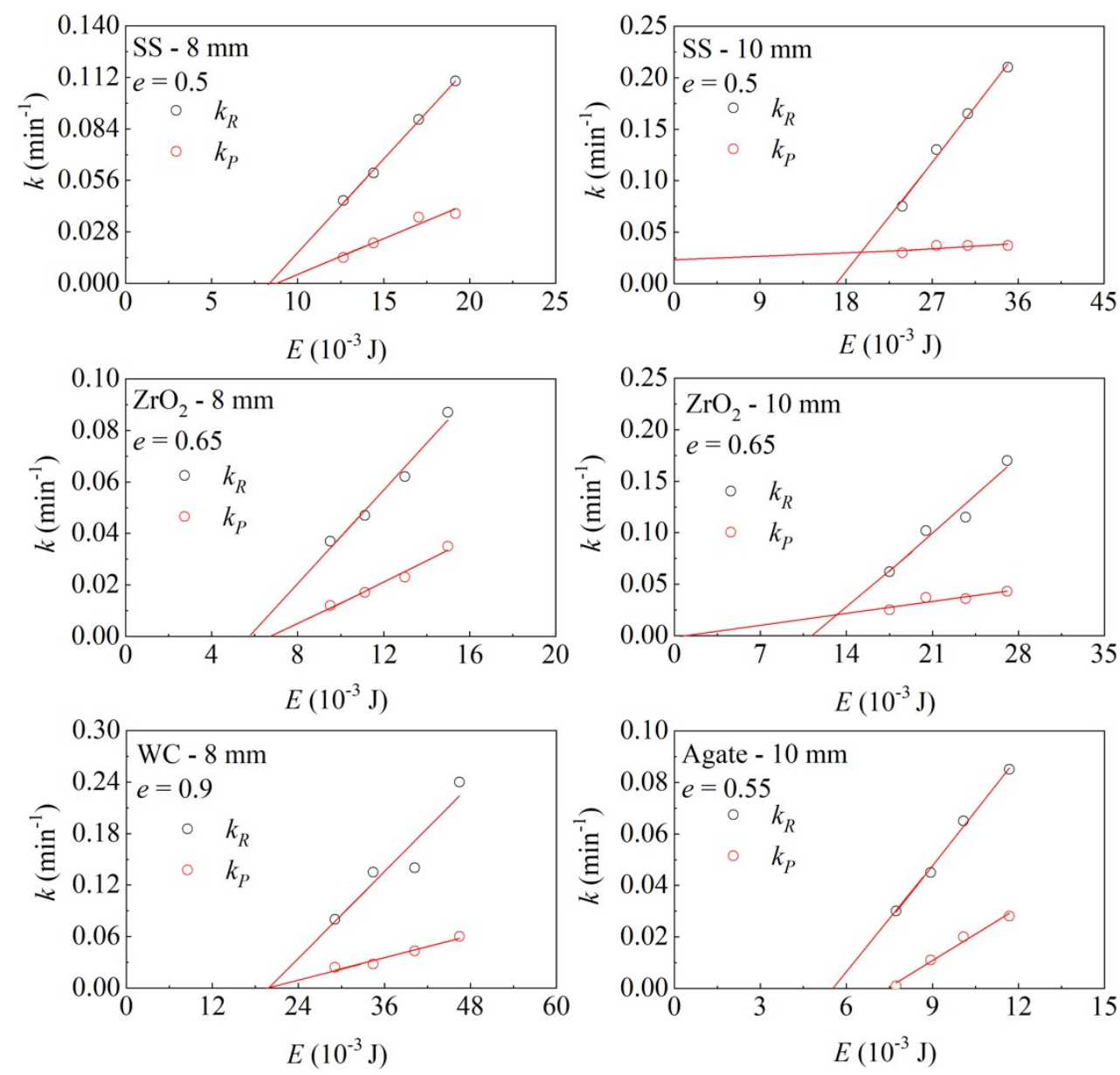

Figure 4 . The $k$ values characteristic of the reactant phase, $k_{R}$, and of the product phase $k_{P}$, as a function of the impact energy. The impact energy was determined in numerical simulations.

Data indicate that $k_{P}$ and $k_{R}$ increase with the impact energy, $E$. The best-fitted lines cross the abscissa at finite $E$ values. These can be interpreted as activation impact energies, hereafter denoted as $E_{0}$. The best-fitted $E_{0}$ are different for different balls, but increase linearly with the ball mass, $m_{\text {ball }}$ (Fig. 5). The ball diameter seems to have a lesser contribution (note the near overlap of two points for balls with the mass of around $1.5 \mathrm{~g}$ ). However, the full explanation as to why the $E_{0}$ values would be different for balls of different masses is, at present, not entirely clear. One reason could be in the different geometry of ball impacts, but a more likely reason could be longer duration of impacts with a heavier ball where the 
deformations of the compressed powder would be more substantial with more of the powder experiencing CLCs upon each impact and resulting in a faster transformation.

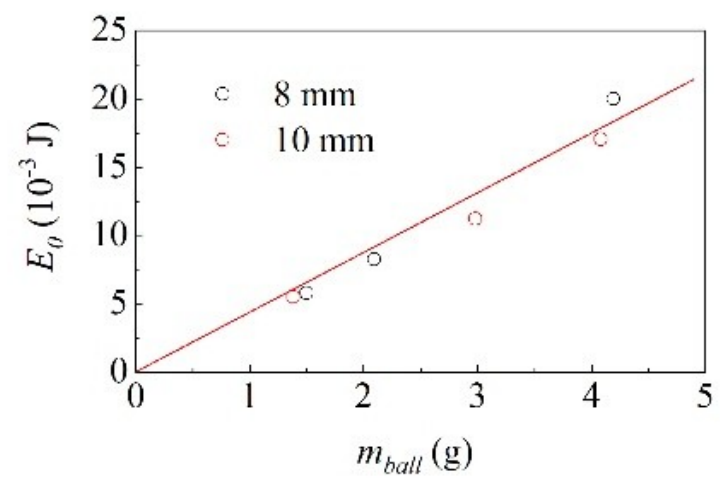

Figure 5. The activation impact energy, $E_{0}$, obtained from the linear change of $k_{R}$, as a function of the ball mass, $m_{\text {ball }}$.

The linear trends for different milling balls can be suitably rescaled by subtracting the energy $E_{0}$ leading to a remarkable overlap of all the reaction profiles when plotted against the rescaled energy $E_{S C}=E-E_{0}$ (Fig. 6). These results suggest that the fraction of impact energy is effectively used to induce the trimerisation and that this is the quantity that critically governs the kinetics of the mechanochemical transformation. Finally, we return to the evaluation of impact energies, which were estimated by numerical simulation using restitution coefficients evaluated on the basis of the experimental kinetic evidence. The restitution coefficients should reflect the hardness of the ball material and indeed, they are showing a linear dependence with the Mohs scale, increasing as the material hardness increase and collisions become more elastic with harder ball materials (Fig. 7). The rate of energy transfer determines reaction kinetics - higher the transfer, faster the reaction - but it stems from conflicting contributions: The more inelastic ball collisions lead to higher energy transfer in individual impacts, but less of the kinetic energy of the ball is restored and consequently, it becomes slower at the next impact and, at a given milling frequency, impacts become less frequent. 


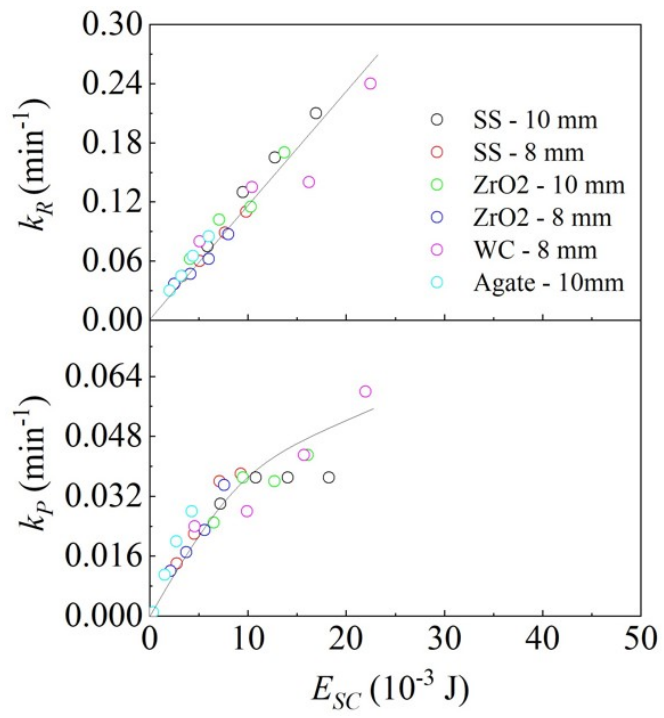

Figure 6 . The $k_{R}$ and $k_{P}$ values as a function of the rescaled energy, $E_{S C}$, for all the milling conditions

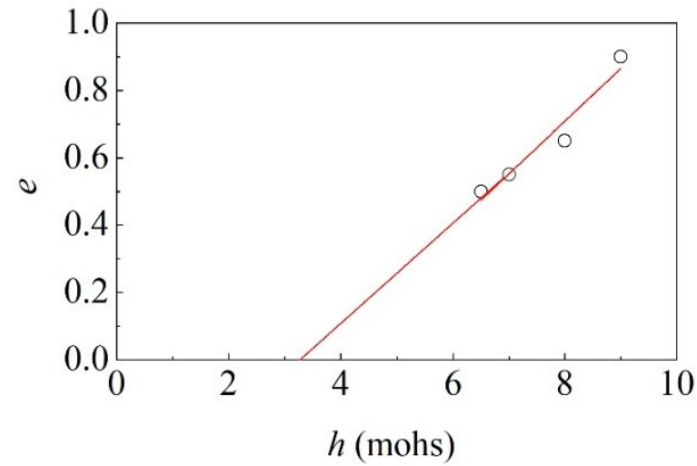

Fig. 7. The restitution coefficient, $e$, used in numerical simulations of milling as a function of the material hardness, $h$.

\section{Conclusions}

Numerical simulations of ball milling and in situ monitoring of a purely mechanically activated reaction, reveal that the mechanochemical reaction rate scales linearly with the energy dose received by the sample. The energy dose in time increases linearly with the third power of the milling frequency meaning that, for example, a 10\% increase in frequency results in approximately $30 \%$ higher energy dose in time. Numerical simulations estimated the average ball velocity and energy transfer in impacts indicating that 
the energy transfer to the sample depends on the hardness of the ball material and we identify an impactenergy threshold below which, at least apparently, no reaction occurs. Next to setting a basis for decoupling mechanically-activated and mixing-driven mechanochemical reactivity, we provide here thorough understanding of energy transfer which will be particularly relevant for "energetically challenging" reactions. ${ }^{16}$ Understanding of energy transfer should also be highly applicable in mechanoredox reactions where reactivity is crucially dependent on the compression of the piezoelectric material. ${ }^{10,11}$ Following his work, we anticipate a wider use of in situ monitoring and kinetic modelling of mechanochemical processes, in particular since both methodologies are now described in technical

detail. $^{29,35,36}$ Finally, we note that the herein studied trimerisation of $\mathrm{Ni}(\mathrm{dbm})_{2}$ could serve as a model reaction, instead of self-sustained reactions, ${ }^{24,37}$ for comparing energy effects of different ball mills.

Acknowledgements. This work is a contribution to the COST (European Cooperation on Science and Technology) Action CA18112 - Mechanochemistry for Sustainable Industry. We thank the Croatian Science Foundation for financing (grant No. 1419). L.V. is supported by the Croatian Science Foundation. M.C. performed her activity within the framework of the International PhD in Innovation Sciences and Technologies at the Università degli Studi di Cagliari, Italy.

\section{References}

1 J.-L. Do and T. Friščić, ACS Cent. Sci., 2017, 3, 13-19.

2 L. Takacs, Chem. Soc. Rev., 2013, 42, 7649-7659.

3 M. Senna, J. Mater. Sci., 2004, 39, 4995-5001.

4 W. Jones and M. D. Eddleston, Faraday Discuss., 2014, 170, 9-34.

5 Y. Zhang, Y. Wang, X. Yang, L. Zhao, R. Su, J. Wu, D. Luo, S. Li, P. Chen, M. Yu, Q. Gong and R. Zhu, Adv. Mater., n/a, 2107420.

6 J. L. Howard, Q. Cao and D. L. Browne, Chem. Sci., 2018, 9, 3080-3094.

7 T. Friščić, C. Mottillo and H. M. Titi, Angew. Chem. Int. Ed., 2020, 59, 1018-1029.

8 K. J. Ardila-Fierro and J. G. Hernández, ChemSusChem, 2021, 14, 2145-2162.

9 T. Friščić and L. Fábián, CrystEngComm, 2009, 11, 743-745.

10 K. Kubota, Y. Pang, A. Miura and H. Ito, Science, 2019, 366, 1500-1504.

11 C. Schumacher, J. G. Hernández and C. Bolm, Angew. Chem., 2020, 132, 16499-16502.

12 F. Gomollón-Bel, Chem. Int., 2019, 41, 12-17.

13 D. E. Crawford, C. K. G. Miskimmin, A. B. Albadarin, G. Walker and S. L. James, Green Chem., 2017, 19, 1507-1518.

14 S. Lukin, M. Tireli, T. Stolar, D. Barišić, M. V. Blanco, M. Di Michiel, K. Užarević and I. Halasz, J. Am. Chem. Soc., 2019, 141, 1212-1216.

15 S. Lukin, I. Lončarić, M. Tireli, T. Stolar, M. V. Blanco, P. Lazić, K. Užarević and I. Halasz, Cryst. Growth Des., 2018, 18, 1539-1547.

16 J. M. Andersen and J. Mack, Chem. Sci., 2017, 8, 5447-5453.

17 E. Gil-González, L. A. Pérez-Maqueda, P. E. Sánchez-Jiménez and A. Perejón, J. Phys. Chem. Lett., 2021, 12, 5540-5546.

18 M. Ferguson, M. S. Moyano, G. A. Tribello, D. E. Crawford, E. M. Bringa, S. L. James, J. Kohanoff and M. G. D. Pópolo, Chem. Sci., 2019, 10, 2924-2929. 
19 L. S. Germann, M. Arhangelskis, M. Etter, R. E. Dinnebier and T. Friščić, Chem. Sci., 2020, 11, 10092-10100.

20 F. Delogu and L. Takacs, J. Mater. Sci., 2018, 53, 13331-13342.

21 A. A. L. Michalchuk, I. A. Tumanov and E. V. Boldyreva, CrystEngComm, 2019, 21, 2174-2179.

22 A. M. Belenguer, A. A. L. Michalchuk, G. I. Lampronti and J. K. M. Sanders, Beilstein J. Org. Chem., 2019, 15, 1226-1235.

23 F. Fischer, N. Fendel, S. Greiser, K. Rademann and F. Emmerling, Org. Process Res. Dev., 2017, 21, 655-659.

24 H. Petersen, S. Reichle, S. Leiting, P. Losch, W. Kersten, T. Rathmann, J. Tseng, M. Etter, W. Schmidt and C. Weidenthaler, Chem. - Eur. J., 2021, 27, 12558-12565.

25 I. Huskić, C. B. Lennox and T. Friščić, Green Chem., 2020, 22, 5881-5901.

26 D. V. Soldatov, A. T. Henegouwen, G. D. Enright, C. I. Ratcliffe and J. A. Ripmeester, Inorg. Chem., 2001, 40, 1626-1636.

27 K. Užarević, N. Ferdelji, T. Mrla, P. A. Julien, B. Halasz, T. Friščić and I. Halasz, Chem. Sci., 2018, 9, 2525-2532.

28 H. Kulla, M. Wilke, F. Fischer, M. Röllig, C. Maierhofer and F. Emmerling, Chem. Commun., 2017, 53, 1664-1667.

29 M. Carta, E. Colacino, F. Delogu and A. Porcheddu, Phys. Chem. Chem. Phys., 2020, 22, 1448914502.

30 E. Colacino, M. Carta, G. Pia, A. Porcheddu, P. C. Ricci and F. Delogu, ACS Omega, 2018, 3, 91969209.

31 A. L. Spek, J. Appl. Crystallogr., 2003, 36, 7-13.

32 S. Lukin, T. Stolar, M. Tireli, M. V. Blanco, D. Babić, T. Friščić, K. Užarević and I. Halasz, Chem. Eur. J., 2017, 23, 13941-13949.

33 S. Garroni, S. Soru, S. Enzo and F. Delogu, Scr. Mater., 2014, 88, 9-12.

34 S. Garroni, S. Enzo and F. Delogu, Scr. Mater., 2014, 83, 49-52.

35 I. Halasz, S. A. J. Kimber, P. J. Beldon, A. M. Belenguer, F. Adams, V. Honkimäki, R. C. Nightingale, R. E. Dinnebier and T. Friščić, Nat. Protoc., 2013, 8, 1718-1729.

36 S. Lukin, K. Užarević and I. Halasz, Nat. Protoc., 2021, 1-40.

37 L. Takacs and V. Šepelák, J. Mater. Sci., 2004, 39, 5487-5489. 